Research letter

\title{
Local immune disbalance correction of the oral cavity as a part of chronic catarrhal gingivitis complex treatment in children
}

\author{
Yury A. Ippolitov, Svetlana A. Garkavets, Tatiana A. Rusanova, Svetlana N. Yudenkova, Victoria P. Kuralesina, \\ Elena O. Aleshina, Yana A. Plotnikova, Chung C. Chan, Evgeni S. Manshin
}

Voronezh N.N.Burdenko State Medical University, Voronezh, Russia

Received 12 April 2016, Revised 9 February 2017, Accepted 5 March 2017

(C) 2016, Ippolitov Y.A., Garkavets S.A., Rusanova T.A., Yudenkova S.N., Kuralesina V.P., Aleshina E.O., Plotnikova Y.A., Chan C.C., Manshin E.S. (C) 2016, Russian Open Medical Journal

\begin{abstract}
Background - Periodontal diseases in adolescents are considered to be of current interest, since the incidence of these conditions tends to increase. Children being in the period of intensive growth are especially exposed to these conditions. Recent studies of molecular morphological disorders occurring in children with chronic catarrhal gingivitis indicate the need of immunocorrectors application in the complex treatment of patients with these disorders, that helps improve both periodontal tissue status and general health.

Material and Methods - 157 children aged 12-15 years were examined. Physical examination of a child included past medical history findings and oral cavity examination. Russian Medical Association (RMA) index was used to diagnose gingivitis; Green-Vermillion index was used to evaluate oral hygiene status.

Results - Changes in the general health of adolescents suffering from periodontal diseases are reported to give evidence of host defense mechanism decrease. Therefore, the treatment is stated to obligatory affect both - the inflammatory process itself and the entire immunity increase. In that case, immunocorrectors application, namely "Imudon", is regarded to be the most reasonable measure. "Imudon" is known to affect the recovery of the regular residential flora in the human oral cavity and has the anti-inflammatory effect as well. "Imudon" application leads to reduction of mucus periodontal pathogens amount. Dissolving in the oral cavity these tablets activate host defense mechanism. Due to excipients active components of this medication remain in the oral cavity for a long-term period.

Conclusion - There was established the correspondence between blood plasma immunological indication changes in children with chronic catarrhal gingivitis receiving "Imudon" therapy before and after treatment.
\end{abstract}

Keywords: gingivitis, adolescents, treatment, immunocorrectors

Cite as Ippolitov YA, Garkavets SA, Rusanova TA, Yudenkova SN, Kuralesina VP, Aleshina EO, Plotnikova YA, Chan CC, Manshin ES. Local immune disbalance correction of the oral cavity as a part of chronic catarrhal gingivitis complex treatment in children. Russian Open Medical Journal 2017 ; 6: e0209.

Correspondence to Yuri A. Ippolitov. Phone: +79050517329. E-mail: dsvgma@mail.ru.

\section{Introduction}

Periodontal diseases are considered to be one of the most common maxillofacial disorders. Incidence of gingivitis is almost $100 \%$, children and young people up to $25-30$ years being affected mostly. Development and implementation of successful treatment methods of periodontal diseases are critical part of national and foreign studies during the last 20 years [1-5]. Microbial factor, which is identified as dental plaque or soft dental deposits in clinical practice, appears to be the main etiological factor of gingivitis. Development of clinical instant testing investigating structure and/ or content of gingival crevice and oral fluid, prediction of remission duration and exacerbation based on microbiological findings, is very important in oral microbiology. Thus, studying of factor microbial homeostasis is of great theoretical and practical significance currently, because it reveals interaction mechanisms between microorganisms and tissues in the oral cavity [2-6]. Chronic gingivitis in children is stated to be of special interest for the prevention and treatment of periodontal diseases. Pharmaceutical efficiency is defined by the fact how fast they can eliminate the causative factor, or make its negative impact impossible, and by the absence of recurrences in children. There exists the problem of insufficient effectiveness of the methods of treatment applied currently. Aspects connected with the chronic catarrhal gingivitis therapy appear to be cross-sectoral issues between dentistry and therapy. Periodontal medicine (the term was first used by Offenbacher in 1998) is regarded as a new section of periodontology, which investigates mutual impact of systemic diseases and inflammatory periodontal diseases to develop new diagnostic criteria, therapy strategies and the integral preventive program of periodontal diseases $[1,4,7,8]$. Recent research studies of molecular morphological disorders arising under chronic catarrhal gingivitis in children detect the necessity of the immunocorrectors' application in the complex therapy of such patients that allows affecting both periodontium and the whole body. 
Table 1. Distribution of children according to the incidence and severity of gingivitis

\begin{tabular}{ccccc}
\hline $\begin{array}{c}\text { Age, } \\
\text { y.o. }\end{array}$ & $\begin{array}{c}\text { Incidence of } \\
\text { ging., no. (\%) }\end{array}$ & $\begin{array}{c}\text { Absence of } \\
\text { ging., no. (\%) }\end{array}$ & $\begin{array}{c}\text { Mild ging., } \\
\text { no. (\%) }\end{array}$ & $\begin{array}{c}\text { Moderate } \\
\text { ging., no. (\%) }\end{array}$ \\
\hline 12 & $15(38.5)$ & $24(61.5)$ & $7(17.9)$ & $8(20.5)$ \\
13 & $26(57.8)$ & $19(42.2)$ & $14(31.1)$ & $12(26.7)$ \\
14 & $24(64.9)$ & $13(35.1)$ & $8(21.6)$ & $16(43.2)$ \\
15 & $28(77.8)$ & $8(22.2)$ & $5(13.9)$ & $23(63.9)$ \\
\hline
\end{tabular}

y.o., years old; ging., gingivitis.

Table 2. Blood plasma IgA, IgG, IgM immunoglobulins test in children before "Imudon" therapy

\begin{tabular}{lccc}
\hline Parameters & $\begin{array}{c}\text { Control } \\
\text { group } \\
(n=31)\end{array}$ & $\begin{array}{c}\text { Children with chronic catarrhal gingivitis } \\
\text { before "Immudon" therapy }(n=62)\end{array}$ \\
\hline $\lg \mathrm{A}, \mu \mathrm{M} / \mathrm{I}$ & $21.3 \pm 0.2$ & $23.7 \pm 0.3^{*}$ & $24.1 \pm 0.4^{*}$ \\
$\lg \mathrm{M}, \mu \mathrm{M} / \mathrm{I}$ & $131.8 \pm 0.1$ & $133.4 \pm 0.2^{*}$ & $135.3 \pm 0.1^{*}$ \\
$\lg \mathrm{M}, \mu \mathrm{M} / \mathrm{I}$ & $20.5 \pm 0.1$ & $19.4 \pm 0.2^{*}$ & $21.3 \pm 0.2^{*}$ \\
\hline
\end{tabular}

Data presented as mean with its standard error ( $\mathrm{M} \pm \mathrm{m})$.

*Statistically significant $(p<0.05)$ differences comparing to healthy children.

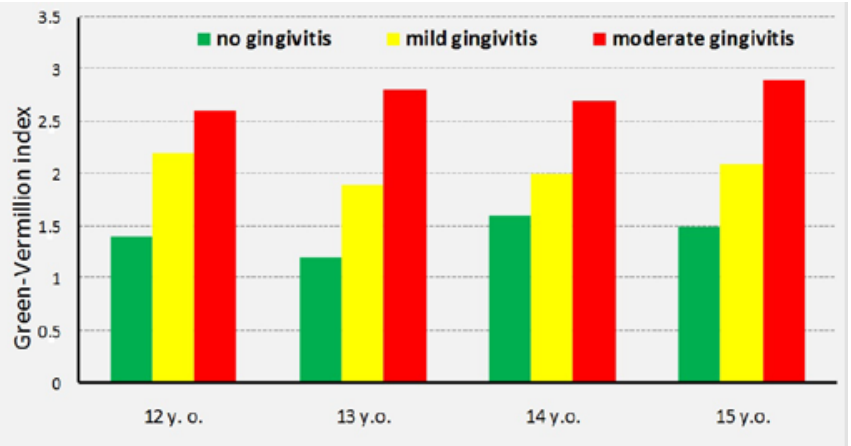

Figure 1. Green-Vermillion index data depending on the age. y.o., years old.

\section{Material and Methods}

\section{Ethical consideration}

All interventions carried out during the research study were approved by the Ethical Committee of the Voronezh N.N.Burdenko State Medical University (Protocol № 5, May 20, 2012).

\section{Patients}

To prove the rationale, the incidence of chronic catarrhal gingivitis in children and the dependence of its severity on the oral hygiene status were investigated. 157 children aged 12-15 were examined. According to clinical findings there were selected 93 children with chronic catarrhal gingivitis (54 patients with mild chronic catarrhal gingivitis and 39 patients with moderate chronic catarrhal gingivitis) (Table 1).

Furthermore, the group of adolescents under investigation with chronic catarrhal gingivitis was divided into two groups: children who underwent the immunocorrectors' therapy (62 children) and children who lacked such a therapy (31 children).

\section{Methods}

Physical examination of a child included past medical history findings and oral cavity examination. Papillary-marginal-alveolar index (PMA) [9] in the modification of Parma (1960) [10] was used to diagnose gingivitis; Green-Vermillion index [11] was used to evaluate oral hygiene status.

Shedding of microorganisms from their natural habitat - oral cavity tissues and fluids - was carried out by the culturing of the materials under investigation on artificial media. The method used is called culture-based investigation. Radial immunodiffusion, or Mancini method [12], was used to reveal changes of plasma immunological indicators in children with chronic catarrhal gingivitis.

\section{Design}

Preventive and curative interventions were performed on the following schedule:

The $1^{\text {st }}$ stage has been reported to be the clinical diagnosis of the disease: evaluation of the oral hygiene status using GreenVermillion index, evaluation of periodontal tissue status using PMA index. Laboratory methods of investigations consisted of microbiological study of the gingival crevice and oral fluid content, and the investigation of plasma immunological indicators in children with chronic catarrhal gingivitis before the application of the immunocorrectors therapy.

The $2^{\text {nd }}$ stage was stated to include complex therapy of chronic catarrhal gingivitis in children using locally immune therapy and the immunocorrector "Imudon". In three months laboratory methods of investigation were performed, i.e. microbiological study of the gingival crevice and oral fluid content, and the investigation of plasma immunological indicators in children with chronic catarrhal gingivitis after the performing of locally immune therapy and the immunocorrector "Imudon" respectively.

\section{Statistical analysis}

Primary data were analyzed with applied Statistics package Statistica 6.0 (Statsoft Inc., USA).

The normality conditions of the data investigated and the dispersion test of signs distribution in the compared groups were assessed using Shapiro-Wilks Test. The value equal 0.05 was decided to become a statistical significance threshold.

We used follow descriptive statistics: minimum and maximum (min-max), mean with its standard error $(\mathrm{M} \pm \mathrm{m})$.

\section{Results and Discussion}

Physical investigation of children was carried out according to the specially developed schedule and the data obtained were listed in the medical history. The dependence of the gingivitis incidence and severity on the oral hygiene status, and the role of the calculus as a factor contributing to etiology of the disease investigated were proved. PMA index was used to diagnose gingivitis, the oral hygiene status was evaluated using GreenVermillion index (Figure 1).

As it is shown on the Figure 1, in chronic mild catarrhal gingivitis the oral hygiene has always been evaluated as unsatisfactory (Green-Vermillion index: 1.9-2.2), in moderate chronic catarrhal gingivitis - as unsatisfactory and bad (GreenVermillion index: 2.5-2.9). The obtained data of the physical examination of the patients show that to increase the 
effectiveness of the professional oral hygiene as a preventive method against inflammatory periodontal diseases is necessary to control oral hygiene and periodontal tissue status every 3-4 months and to perform preventive measures in the amount essential to each patient. To evaluate local immunity in children with chronic catarrhal gingivitis single radial immunodiffusion assay, or Mancini method, was applied. Fasting blood sampling was done in the morning. Blood plasma $\lg A$, IgG, IgM immunoglobulins were tested by Mancini method in children of both groups - the main and control. This investigation was performed twice: before and after the preventive and curative interventions. Findings of children with chronic catarrhal gingivitis were abnormal with increased blood plasma immunoglobulin level. The highest findings were observed in children with moderate gingivitis comparing to children with mild gingivitis (Table 2). Findings of the children in the control group were similar to those in the main group. Blood plasma IgA, IgG, IgM immunoglobulins test was repeated three months after the beginning of "Imudon" therapy in patients of the main group and in children of the control group receiving conventional treatment.

Immunoglobulins test findings in children receiving "Imudon" therapy improved considerably comparing to children being treated conventionally (Table 3 ).

Children of the control group receiving conventional therapy are noted to also manifest improved immunoglobulin findings slightly. After the application of the locally immune therapy in adolescents with chronic catarrhal gingivitis, both - oral hygiene status and inflammation intensity improved (Figure 2).

Apparently, the average inflammation intensity (PMA index) in all adolescents constitutes $24.30 \pm 1.16 \%$ (Green-Vermillion index: $2.60 \pm 0.06)$ at the first stage, the average inflammation intensity (PMA index) during the application of the "Imudon" immunocorrector constitutes $10.40 \pm 0.43 \%$ (Green-Vermillion index: $1.70 \pm 0.05)$ at the second stage; and three months after the "Imudon" therapy the average inflammation intensity (PMA index) in all adolescents constitutes $1.80 \pm 0.24 \%$ (Green-Vermillion index: $0.90 \pm 0.05$ ) respectively.

Oral fluid and gingival crevicular fluid microbiological tests performed in adolescents with chronic catarrhal gingivitis before treatment revealed the following main types of microorganisms: streptococci, staphylococci, micrococcaceae, Neisseria, Corynebacterium spesits, Enterodacter species, Pseudomonas species, and Candida albicans. The most common (with the incidence $21 \%$ and more) were $\beta$-hemolytic Streptococci, Neisseria, fungi Candida albicans.

Quantitative microbiological findings of Streptococci, Staphylococci, Micrococcaceae, Neisseria, Corynebacterium spesits, Enterodacter species, Pseudomonas species, and Candida albicans revealed the most valid decrease after the professional oral hygiene similar to clinical research findings. Thus, the dynamics of $\beta$-hemolytic Streptococci, Neisseria, Candida albicans fungi was analyzed three months after the locally immune therapy in adolescents with chronic catarrhal gingivitis.

Changes in the average $\beta$-hemolytic Streptococci colonies amount and the amount of their detection in both - oral and gingival crevicular fluids, depends directly on the oral hygiene status; in other words, improvements of the oral hygiene status lead to decrease in the average amount of $\beta$-hemolytic streptococci colonies amount in oral and gingival crevicular fluids.
Table 3. Blood plasma IgA, IgG, IgM immunoglobulins test in children after "Imudon" therapy

\begin{tabular}{lccc}
\hline Parameters & $\begin{array}{c}\text { Control } \\
\text { group } \\
(n=31)\end{array}$ & $\begin{array}{c}\text { Children with chronic catarrhal gingivitis } \\
\text { after "Immudon" therapy }(n=62)\end{array}$ \\
\hline $\lg \mathrm{A}, \mu \mathrm{M} / \mathrm{I}$ & $17.3 \pm 0.2$ & $10.7 \pm 0.2^{*}$ & $13.5 \pm 0.3^{*}$ \\
$\lg , \mu \mathrm{M} / \mathrm{I}$ & $116.8 \pm 0.1$ & $79.8 \pm 0.1^{*}$ & $82.2 \pm 0.1^{*}$ \\
$\lg \mathrm{M}, \mu \mathrm{M} / \mathrm{I}$ & $14.5 \pm 0.1$ & $7.5 \pm 0.1^{*}$ & $9.3 \pm 0.1^{*}$ \\
\hline
\end{tabular}

Data presented as mean with its standard error $(\mathrm{M} \pm \mathrm{m})$.

*Statistically significant $(p<0.05)$ differences comparing to healthy children.

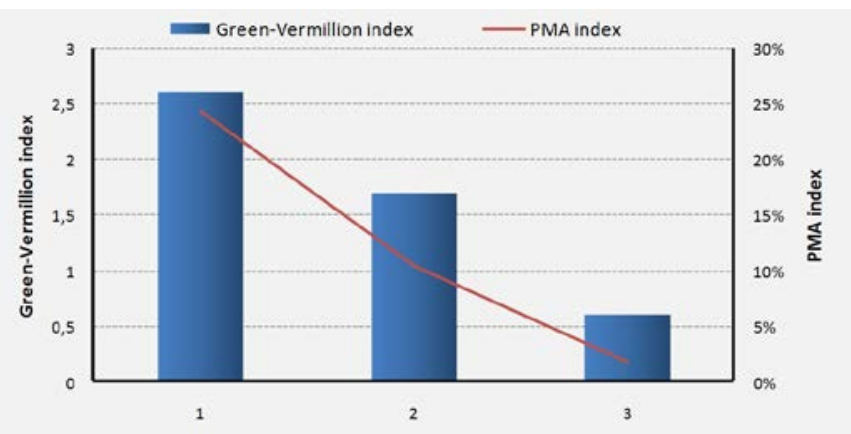

Figure 2. PMA index and Green-Vermillion index average findings depending on the therapy stage.

Consequently, possible $\beta$-hemolytic Streptococci implication in the dental plaque development is proved. Quantitative Neisseria and Candida albicans microbiological findings in the oral and gingival crevicular fluids were also reported to change. The abovementioned findings decreased significantly after the locally immune therapy and professional oral hygiene, as well as the oral hygiene status correction, in children of the control group. In such a way, the average amount of Neisseria colonies decreased 13.6 times in the oral fluid, 32 times - in the gingival crevicular fluid. The decrease of Neisseria and Candida albicans colonies amount appears to reduce the gingival inflammation intensity in children with mild or moderate chronic catarrhal gingivitis.

As can be seen from the above, after the professional oral hygiene and oral hygiene status correction the improvements of oral status and decrease in inflammation intensity led to changes in microbiological findings of the microorganisms under investigation in the adolescents with chronic catarrhal gingivitis. Body changes in adolescents suffering from the periodontal diseases give evidence of the natural protective mechanisms reduction. Due to this, the treatment must be targeted not only at the elimination of inflammation process but at the immunity improvements as well. Then the application of immunocorrectors would be reasonable. These medications are regarded to activate oral mucosa defense providing the increase amount of a special enzyme presenting in the saliva - lysozyme that is known to be microbicidically active, stimulating and increasing the number of immunocompetitive cells responsible for antibodies production. "Imudon" is considered to be such an immunocorrector. "Imudon" application causes the recovery of the resident microfloras found in the oral cavity and anti-inflammatory effect. "Imudon" administration leads to the decrease amount of pathogenic microorganisms on the periodontal mucosa. Being dissolved in the oral cavity these tablets activate the body natural immune defense. Due to excipients active components affect the oral 
mucosa for a long period. Considering all the above mentioned we can conclude that application of "Imudon" as a locally immune therapy component gives positive effect in complex treatment of the chronic catarrhal gingivitis in adolescents.

\section{Conclusion}

The incidence of chronic catarrhal gingivitis in children aged $12-15$ years is still rather high and constitutes $76.3-86.9 \%$.

There was established the correspondence between blood plasma immunological indication changes in children with chronic catarrhal gingivitis receiving "Imudon" therapy before and after treatment.

Age-related quantitative changes of $\beta$-hemolytic Streptococci indications in the oral and gingival crevicular fluids in children with chronic catarrhal gingivitis were similar to those obtained after the "Imudon" therapy in the research dynamics. Quantitative indications of Neisseria and Candida albicans in the oral and gingival crevicular fluids reduced gradually going with the dynamics of the inflammation intensity changes (PMA index).

Conflict of interest: none declared.

\section{References}

1. Albitskaya YN. Peculiarities of biochemical changes in the oral cavity in the young suffering from caries and gingivitis depending on the age and the season: PhD thesis. Rostov-na-Donu, Russia, 2005. Russian

2. Ulitovskiy S. Distribution of personal oral hygiene facilities and their role in the prevention of dental disorders. DentArt 2004; (1): 21. Russian

3. Ippolitov YA. Complex chronic generalized gingivitis therapy with betatherapy application: PhD dissertation. Voronezh, Russia, 1996; 256 p. Russian

4. Garkavets SA. Risk factors, homeostasis, intensity and incidence of caries in children: PhD dissertation. Voronezh, Russia, 2008; 232 p. Russian

5. Kuralesina VP. The role of the oral professional hygiene in chronic gingivitis therapy and prevention: PhD dissertation. Voronezh, Russia, 2003; 122 p. Russian

6. Tsepov LM, Nikolaev AI, ZHazhnov EN. On etiology and pathogenesis of inflammatory periodontal diseases (review). Parodontologiya 2000; (2): 9-12. Russian

7. Kameron A, Uidmer R, Vinogradova TF. Reference book on pediatric dentistry. Moscow, Russia: MEDpress-inform, 2003. Russian

8. Petrovich YA, Puzin MN, Sukhova GV. Free-radical oxidation and saliva and blood anti-oxidant defense in chronic generalized paradontitis. Rossiyskiy Stomatologicheskiy Zhurnal 2000; (3): 11-13. Russian

9. Schour J, Massler M. Gingval disease in postwar Italy (1945). J Am Dent Assoc 1947; 35: 475-482. https://www.ncbi.nlm.nih.gov/pubmed/20260900.

10. Parma C. Parodontopathien. Leipzig: JA Barth, 1960; 203 p. German

11. Green JC, Vermillion JR. The oral hygiene index: a method for classifying oral hygiene status. J Am Dent Assoc 1960; 61: 172-179. http://dx.doi.org/10.14219/jada.archive.1960.0177.

12. Mancini G, Carbonara AO, Heremans JF. Immunochemical quantitation of antigens by single radial immunodiffusion. Immunochemistry 1965; 2(3): 235-254. http://dx.doi.org/10.1016/0019-2791(65)90004-2.

\section{Authors:}

Yury A. Ippolitov - MD, DSc, Associate Professor, Head of the Pediatric Dentistry and Orthodontics Chair, Voronezh N.N. Burdenko State Medical University, Voronezh, Russia. http://orcid.org/0000-0001-9922-137X.
Svetlana A. Garkavets - MD, PhD, Assistant, Pediatric Dentistry and Orthodontics Chair, Voronezh N.N. Burdenko State Medical University, Voronezh, Russia.

Tatiana A. Rusanova - MD, PhD, Assistant, Pediatric Dentistry and Orthodontics Chair, Voronezh N.N. Burdenko State Medical University, Voronezh, Russia.

Svetlana N. Yudenkova - MD, PhD, Associate Professor, Pediatric Dentistry and Orthodontics Chair, Voronezh N.N. Burdenko State Medical University, Voronezh, Russia.

Victoria P. Kuralesina - MD, PhD, Associate Professor, Pediatric Dentistry and Orthodontics Chair, Voronezh N.N. Burdenko State Medical University, Voronezh, Russia.

Elena O. Aleshina - MD, Assistant, Pediatric Dentistry and Orthodontics Chair, Voronezh N.N. Burdenko State Medical University, Voronezh, Russia. Yana A. Plotnikova - Postgraduate student, Pediatric Dentistry and Orthodontics Chair, Voronezh N.N. Burdenko State Medical University, Voronezh, Russia.

Chung C. Chan - Postgraduate student, Pediatric Dentistry and Orthodontics Chair, Voronezh N.N. Burdenko State Medical University, Voronezh, Russia.

Evgeni S. Manshin - Residency student, Pediatric Dentistry and Orthodontics Chair, Voronezh N.N. Burdenko State Medical University, Voronezh, Russia. 